\title{
POLICONSUMO SIMULTÁNEO DE DROGAS EN ESTUDIANTES DE FACULTADES DE CIENCIAS DE LA SALUD/CIENCIAS MÉDICAS EN SIETE UNIVERSIDADES DE CINCO PAIISES DE AMÉRICA LATINA Y UN PAÍS DEL CARIBE: IMPLICACIONES DE GÉNERO, LEGALES Y SOCIALES
}

\author{
Andrés Herrera Rodríguez", Rosibel Prieto Silva², Monica Veloza Gomez ${ }^{3}$, Giselle Riquelme Hernández", \\ Maria do Perpétuo Socorro de Sousa Nóbrega ${ }^{5}$, Carole Mitchell ${ }^{6}$, Fabio Bautista Pérez ${ }^{7}$, Joy Harrison ${ }^{8}$, Patrice \\ Whitehorne-Smith ${ }^{9}$
}

${ }^{1}$ Ph.D. Investigador del Centro de Investigación en Demografía y Salud de la Universidad de Nicaragua. Profesor Titular de la Facultad de Ciencias Médicas de la Universidad de Nicaragua. León, Nicaragua. E-mail: andres.herrera@unanleon.edu.ni 2 MSc. Profesora Asociada de la Universidad Nacional de Colombia. Colombia. E-mail: rosibps@gmail.com

${ }^{3}$ MSc. Profesora Asistente del Programa de Enfermeria de la Facultad de Enfermería y Rehabilitacion Universidad de La Sabana. Colombia. E-mail: monica.veloza@unisabana.edu.co

${ }^{4}$ MSc. Profesora Asistente Adjunta del Departamento de Salud de la Mujer de la Escuela de Enfermeria de la Pontificia Universidad Católica de Chile. Chile. E-mail: gfriquel@uc.cl

${ }^{5}$ Ph.D. Investigadora da Fundação Universitária do ABC. São Paulo, Brasil. E-mail: perpetuasn1@gmail.com

${ }^{6}$ Ph.D. Investigadora de la University The West Indies. Jamaica. E-mail: caroledmitchell@gmail.com

${ }^{7}$ MSc. Investigador del Centro de Investigación en Salud y Ciencias Sociales de la Universidad Evangélica de El Salvador. El Salvador. E-mail: fabio.bautista@uee.edu.sv

${ }^{8}$ MSc. Investigadora de la University of The West Indies. Jamaica. E-mail: jarraisjr@gmail.com

${ }^{9}$ MSc. Investigadora de la University The West Indies. Jamaica. E-mail: limpz14@yahoo.com

RESUMEN: El objetivo de este estudio fue comprender los patrones de policonsumo simultáneo de sustancias psicoactivas y sus implicaciones de género, legales y sociales, en estudiantes de primer y segundo año de las facultades de ciencias de la salud/ciencias médicas, en siete universidades de cinco países latinoamericanos, Colombia, Nicaragua, Chile, Brasil y El Salvador, y un país caribeño, Jamaica. El diseño fue un corte transversal. Las combinaciones de alcohol + tabaco y de alcohol + marihuana fueron las mas reportadas en todas las universidades, a excepción de alcohol + tabaco en Jamaica. Los factores asociados al policonsumo más referidos fueron "tener relaciones sexuales inesperada" en la universidad de Brasil, "tener relaciones sexuales sin protección" en las universidades de Chile, Colombia y Nicaragua, "tener problemas con su pareja sentimental" en la universidad de Jamaica, y "ausentarse de clases" en la universidad de El Salvador. Tres entornos se relacionaron, de manera positiva o negativa, con el policonsumo simultáneo de sustancias psicoactivas: estudiantil, familiar y de comportamiento sexual.

DESCRIPTORES: Estudiantes. Conducta adictiva. Transtornos relacionados con sustancias. Factores de riesgo. Drogas ilícitas. América Latina. Caribe.

\section{SIMULTANEOUS POLYDRUG CONSUPTION AMONG UNDERGRADUATE STUDENTS OF HEALTH SCIENCES OF SEVEN UNIVERSITIES IN FIVE LATIN AMERICAN COUNTRIES AND ONE CARIBEAN COUNTRY: GENDER, LEGAL AND SOCIAL IMPLICACIONS}

\begin{abstract}
The objective of this study was to understand patterns of simultaneous polydrug use of psychoactive substances and its gender, legal and social implications, among first and second year undergraduate students in health sciences/medical sciences faculties from six universities in five Latin American countries, Colombia, Nicaragua, Chile, Brazil, and El Salvador, and one Caribbean country, Jamaica. The study design was cross-sectional. The combinations of alcohol + tobacco and alchol + cannabis were the most frequently reported in all universities, with the exception of alcohol + tabaco in Jamaica. The factors associated to polidrug use most frequently mentioned were "to have unexpected sexual relationships" in the Brazilian university, "to have unprotected sex" in the universities of Chile, Colombia and Nicaragua, "to have problems with his o her sentimental partner" in the Jamaican university, and "to skip classes" in the university of El Salvador. Three environments were related, positively or negatively, with simultaneous polydrug use of psychoactive substances: academic, familiar and sexual behavioral.
\end{abstract}

DESCRIPTORS: Students. Behavior, addictive. Substance-related disorders. Risk factors. Street drugs. América Latina. Caribe. 


\title{
POLICONSUMO SIMULTÂNEO DE DROGAS ENTRE ESTUDANTES DE GRADUAÇÃO DE CIÊNCIAS DA SAÚDE DE SETE UNIVERSIDADES EM CINCO PAÍSES DA AMÉRICA LATINA E UM PAÍS DO CARIBE: IMPLICAÇÕES DE GÊNERO, LEGAL E SOCIAIS
}

\begin{abstract}
RESUMO: Estudo de corte transversal que teve por objetivo compreender os padrões de policonsumo simultâneo de substâncias psicoativas e suas implicações de gênero, direitos legais e sociais entre estudantes do primeiro e segundo ano de faculdades de ciencias da saúde/médicas, de sete universidades, em cinco países latinoamericanos e um do Caribe. Os resultados indicam que o uso de múltiplas drogas simultâneas como álcool + tabaco foi maior para as universidades na Colômbia, Nicarágua, Chile, Brasil e El Salvador, com exceção da Jamaica, seguido por policonsumo simultâneo de álcool + maconha. A consequencia mais comum entre os estudantes que usam mais de uma substância psicoativa é o "sexo eventual" na Universidade do Brasil; "ter relações sexuais sem proteção" nas Universidades do Chile, Colômbia e Nicarágua; "ter problemas com seu parceiro sentimental" na Universidade da Jamaica, e ausentar-se das aulas" para a Universidade de El Salvador. Conclui-se que os modelos representam a existência de três ambientes relacionados entre si, positiva ou negativamente, com o uso simultâneo de substâncias psicoativas: ou seja, estudante, família do aluno e comportamento sexual.
\end{abstract}

DESCRITORES: Estudantes. Comportamento aditivo. Transtornos relacionados ao uso de substancias. Fatores de risco. Drogas ilícitas. América Latina. Caribe.

\section{INTRODUCCIÓN}

En America Latina y El Caribe hay preocupación por los estudiantes de universidades por que cada vez son más vulnerables hacia el consumo de drogas. Asimismo, el policonsumo simultáneo de drogas tiene implicaciones legales y sociales para los estudiantes y sus familiares más cercanos. Los estudiantes universitarios son una población vulnerable. ${ }^{1-2}$ La transición de la escuela a la universidad puede ser una experiencia muy agotadora. La nueva experiencia para algunos estudiantes que viven sólos, con poco control parental, los coloca en alto riesgo de uso y/o abuso de sustancias. Además, la universidad es a menudo una cultura de numerosas oportunidades y a veces en los campus universitarios se promueve el consumo de alcohol y otras sustancias como un método para aliviar tensión y para la diversión. ${ }^{3}$ La literatura en Latinoamerica y el Caribe reporta algunas cifras de consumo. En Colombia el alcohol sería la sustancia más consumida 70 y el $90 \%{ }^{4}$ En Chile, cifras indican que el alcohol es la sustancia más usada, con un predominio del $83.5 \%$, seguido del tabaco el $67.4 \%$, marihuana el $16.7 \%$, benzodiazepinas el $7.8 \%$ y cocaína el $3.0 \% .^{5}$ Mientras en El Salvador las sustancias más consumidas son el alcohol y tabaco con $24.8 \%$ y el $19.2 \%$ entre hombres y mujeres. ${ }^{6}$ En Jamaica, para el 2006, el consumo de alcohol entre los estudiantes de secundaria y universtiarios fue de $71.1 \%$, el $1.4 \%$ para alucinógenos y Opio. ${ }^{7}$ En Nicaragua el predominio de vida de abuso de cocaína entre personas entre $12-65$ años es estimado en $2.5 \%$, éxtasis, el $0.2 \%$, marihuana el $7.91 \%$, crack $1.29 \%$ y otras drogas ilícitas el $8.8 \% .{ }^{8}$ Otro estudio realizado por la Organización Panamericana de la Salud en 2006 sobre el consumo de alcohol en los últimos 12 meses reportó el 30\% entre jóvenes (18-24 años).
El presente estudio sigue los principios de la Teoría Bio-psico-social. ${ }^{9-10}$ El policonsumo simultáneo se divide en dos tipos: simultáneo y concurrente. El consumo simultáneo de drogas es la co-ingestión de drogas diferentes al mismo tiempo, y concurrentes es el uso de drogas diferentes pero en diferentes ocasiones. ${ }^{11}$ En un estudio analizado, el policonsumo simultaneo de alcohol y medicamentos de prescripción fue reportado por el $12.1 \%$ de la muestra. Los motivos citados para el consumo fueron: mejoran ciertos efectos deseados, disminuyen efectos no deseados, o cuando se combina regulan los efectos de uno y de otra droga. ${ }^{12}$

En el presente estudio, exploramos el problema de policonsumo simultaneo enfocándonos en la siguiente pregunta: ¿Cuáles son los patrones de policonsumo simultáneo de drogas y las implicaciones de género, sociales y legales en los estudiantes universitarios de pregrado de primer y segundo año de Facultades de Ciencias de la Salud/Ciencias Médicas, en siete universidades de cinco países de América Latina y un país del Caribe? El objetivo fue observar el patrón de policonsumo simultáneo de drogas y sus implicaciones de género, legales y sociales entre estudiante de pregrado de ciencias de la salud/médicas siete universidades en cinco países latinoamericanos y en un país caribeño.

\section{MÉTODOLOGÍA}

Este fue un estudio multicéntrico en siete universidades, tres públicas y cuatro privadas, en los países de Brasil, Colombia, Chile, El Salvador, Nicaragua y Jamaica. El diseño del estudio fue de corte transversal. ${ }^{13}$ Los participantes fueron seleccionados de seis universidades Latinoamericanas (una de Santiago, Chile; dos de Cundinamarca, Co- 
lombia; una de Santo Andre, Brazil; una de León, Nicaragua; una de San Salvador, El Salvador) y una del Caribe (Kingston, Jamaica).

Los participantes fueron estudiantes de pregrado de jornada completa y de media jornada cursando el primer y segundo año en carreras de Ciencias de Salud. El cuestionario estuvo compuesto por cuatro secciones, con 58 preguntas cerradas, las que permitían al entrevistado proporcionar información adicional en caso necesario. El tiempo de aplicación de la encuentra fue de aproximadamente 30 minutos. Para permitir a todos los estudiantes tener las misma chance de participar, los cursos seleccionados para aplicar la encuenta fuereon elegidos al azar.

El análisis de datos incluyó estadísticas descriptivas, para lo cual se utilizó el programa estadístico SPSS, versión 15.0.
Se obtuvo aprobación ética del Comité de Ética de Investigación del Centro de Adicciones y Salud Mental (Centre for Adictions and Mental Health, CAMH), en Ontario, Canadá; así como en cada una de las siete universidades participantes. ${ }^{14}$

\section{RESULTADOS}

\section{Datos generales}

El tamaño total de la muestra agregada fue de 2876 estudiantes de pregrado en siete universidades (ver Tabla 1). Los participantes fueron predominantemente de sexo femenino para todas las univeridades. Las medianas de edad fluctuaron entre 19 y 21 años dependiendo de la universidad.

Tabla 1 - Población universitaria y muestra, según sexo y edad, por paises, 2009-2010

\begin{tabular}{|c|c|c|c|c|c|c|c|c|}
\hline \multirow{3}{*}{ Universidades } & \multirow{3}{*}{$\begin{array}{l}\text { Población } 1^{\circ} \& \\
2^{\circ} \text { Año }(n)\end{array}$} & \multirow{3}{*}{$\begin{array}{l}\text { Muestra } \\
\text { (n) }\end{array}$} & \multicolumn{2}{|c|}{ Edad } & \multicolumn{4}{|c|}{ Sexo } \\
\hline & & & \multirow{2}{*}{ Media } & \multirow{2}{*}{ Mediana } & \multicolumn{2}{|c|}{ M } & \multicolumn{2}{|c|}{$\mathbf{F}$} \\
\hline & & & & & $\mathbf{n}$ & $\%$ & $\mathbf{n}$ & $\%$ \\
\hline $\begin{array}{l}\text { Universidad X en Santo André, } \\
\text { Brazil }\end{array}$ & 523 & 275 & 22.2 & 21 & 53 & 19.3 & 222 & 80.7 \\
\hline Universidad $\mathrm{X}$ en Santiago, Chile & 433 & 188 & 20.1 & 20 & 49 & 26.1 & 139 & 73.9 \\
\hline $\begin{array}{l}\text { Universidades X en Cudinamarca, } \\
\text { Colombia }\end{array}$ & 4100 & 1452 & 20.5 & 20 & 421 & 29.1 & 1028 & 73.9 \\
\hline $\begin{array}{l}\text { Universidades X en León, } \\
\text { Nicaragua }\end{array}$ & 1750 & 357 & 19.5 & 19 & 116 & 32.6 & 240 & 67.4 \\
\hline $\begin{array}{l}\text { Universidad X en San Salvador, } \\
\text { El Salvador }\end{array}$ & 1800 & 309 & 19.5 & 19 & 59 & 19.1 & 250 & 80.9 \\
\hline $\begin{array}{l}\text { Universidad X en Kingston, } \\
\text { Jamaica }\end{array}$ & 1800 & 295 & 29.8 & 21 & 58 & 19.7 & 237 & 80.3 \\
\hline Población total/muestra & 10396 & 2876 & & & & & & \\
\hline
\end{tabular}

Uso de sustancias psicoactivas en los últimos 12 meses

Los porcentajes de respuesta positiva al uso de sustancias psicoactivas en los últimos 12 meses (ver Tabla 2), por país y en orden decreciente, fueron los siguientes: Colombia, $66.5 \%$; Brasil, $65.1 \%$; Chile, $51.1 \%$; Nicaragua, $42.6 \%$; Jamaica, $29.2 \%$; y El Salvador, $18.8 \%$.

Tabla 2 - Uso de sustancias psicoactivas y policonsumo simultaneo de sustancias psicoactivas entre estudiantes de pregrado en los últimos 12 meses y últimos 30 días, por paises, 2009-2010

\begin{tabular}{|c|c|c|c|c|c|c|}
\hline \multirow{3}{*}{ País/lugar } & \multirow{2}{*}{\multicolumn{2}{|c|}{$\begin{array}{l}\text { Uso de sustancias en } \\
\text { los últimos } 12 \text { meses }\end{array}$}} & \multicolumn{4}{|c|}{$\begin{array}{l}\text { Uso de más de una sustancia psicoactiva a } \\
\text { mismo tiempo }\end{array}$} \\
\hline & & & \multicolumn{2}{|c|}{ últimos 12 meses } & \multicolumn{2}{|c|}{ últimos 30 días } \\
\hline & $\mathbf{n}$ & $\%$ & $\mathbf{n}$ & $\%$ & $\mathbf{n}$ & $\%$ \\
\hline Brasil/Santo André (n=275) & 179 & 65.1 & 50 & 27.9 & 30 & 16.8 \\
\hline Chile/Santiago $(n=188)$ & 96 & 51.1 & 41 & 42.7 & 30 & 31.3 \\
\hline Colombia/Cundinamarca $(n=1452)$ & 965 & 66.5 & 326 & 33.8 & 223 & 23.1 \\
\hline El Salvador/San Salvador $(n=309)$ & 58 & 18.8 & 23 & 39.7 & 21 & 36.2 \\
\hline Jamaica/Kingston $(n=195)$ & 86 & 29.2 & 16 & 18.6 & 8 & 9.3 \\
\hline Nicaragua/León $(n=357)$ & 152 & 42.6 & 48 & 31.6 & 34 & 22.3 \\
\hline
\end{tabular}

Texto Contexto Enferm, Florianópolis, 2012; 21 (Esp): 17-24. 


\section{Policonsumo simultáneo}

Respecto a las cifras repoortadas de policonsumo simultaneo en los últimos 12 meses, los mayores porcentajes los presentaron Chile $42.7 \%$ y El Salvador $39.7 \%$, siendo el más bajo el reportado en Jamaica $18.6 \%$. En cuanto al policonsumo en los últimos 30 días, los porcentaje dereporte mas altos los presentaron El Salvador $36.2 \%$ y Chile $31.3 \%$, siendo nuevamente Jamaica el mas bajo $9.3 \%$.

La tabla 3 muestra las cifras de policonsumo simultáneo según combinación de drogas, con porcentajes calculados entre los estudiantes que reportaron policonsumo simultaneo. El patrón de policonsumo mas referido para los últimos 12 meses en todos los países fue el alcohol + tabaco, con excepción de Jamaica donde el más frecuente fue alcohol + marihuana. Los mayores porcentajes de consumo simultáneo de alcohol + tabaco se observan, en orden decreciente, en Colombia, Nicaragua y Chile, con valores cercanos al 50\%. Llaman la atención los altos porcentajes de Brasil en el consumo de alcohol + drogas de prescripción $22 \%$, y de Chile y Brasil en el policonsumo de alcohol con marihuana, y de ambos combinadas con tabaco. La combinación alcohol + tabaco es nuevamente la mas mencionada para policonsumo en los últimos 30 días, con la excepción nuevamente de Jamaica, donde el patrón principal es alcohol + marihuana $\mathrm{y}$ alcohol + tabaco + marihuana. Los mayores porcentajes de consumo simultáneo de alcohol + tabaco para esta categoría se observaron en orden decreciente, en Colombia, Chile, Nicaragua, Brasil y El Salvador. Nuevamente el consumo de alcohol + drogas de prescripción, alcohol y marihuana, y de estas dos últimas con tabaco es alto en Brasil.

Tabla 3 - Cifras de policonsumo simultáneo según combinación de drogas, entre estudiantes de pregrado en los últimos 12 meses y últimos 30 días, por países, 2009-2010

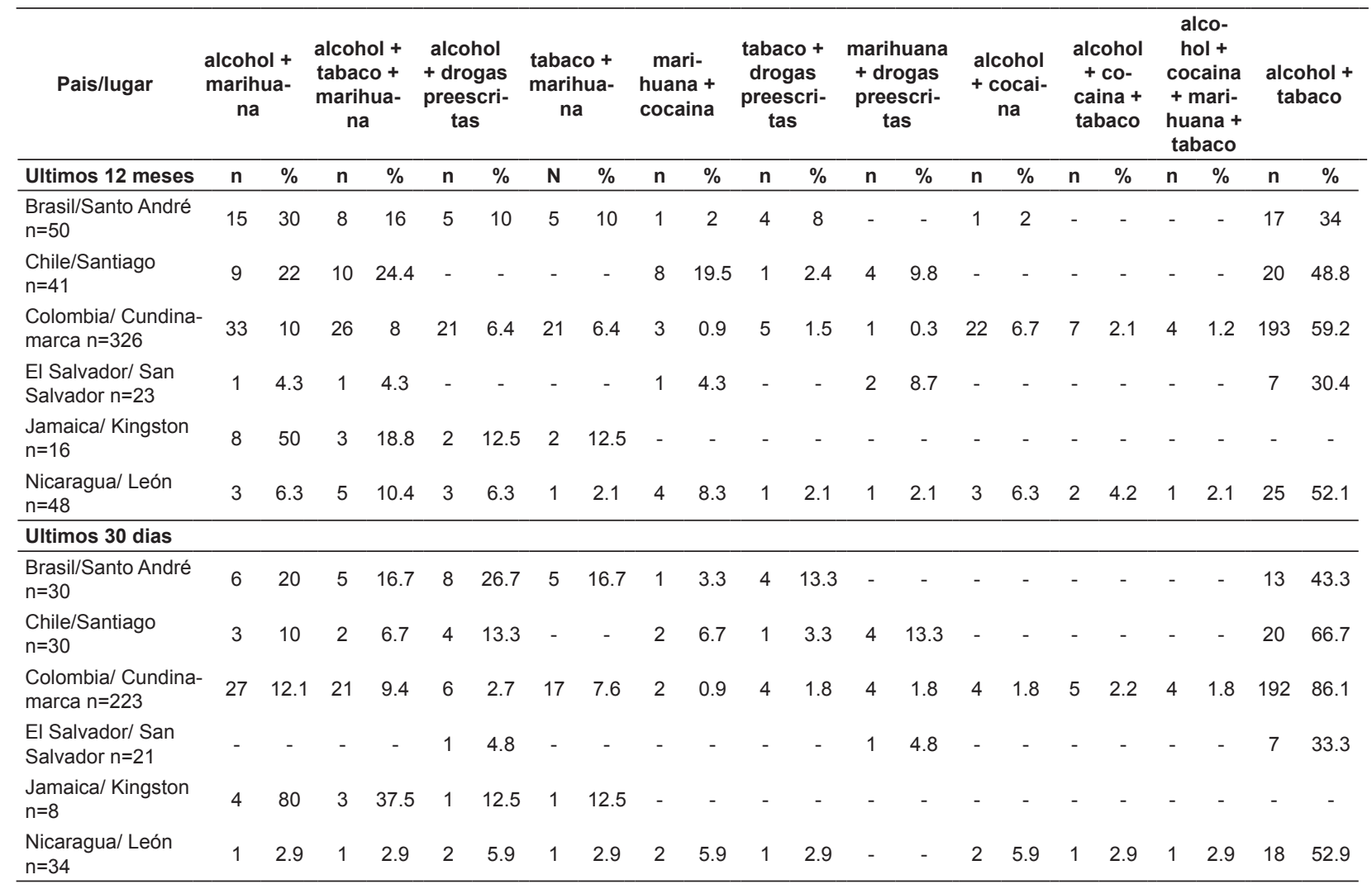

Implicaciones de género y el policonsumo simultaneo de sustancias psicoactivas

En Nicaragua los encuestados respondieron con mayor frecuencia que los hombres influencian a las mujeres en el policonsumo simultáneo $60.4 \%$, afirmación que tuvo la menor cifra de respuesta positiva en Chile $17.1 \%$. En cuanto a la influencia de mujeres sobre hombres, El Salvador presentó la cifra mayor $78.3 \%$ y Chile nuevamente la menor $12.2 \%$, (datos no mostrados). 
Implicaciones sociales y el policonsumo simultaneo de sustancias psicoactivas

La tabla 4 muestra el aspecto social asociado el policonsumo simultáneo de sutancias psicoactivas. La razón "le ayudan a disfrutar la compañía de sus amigos" mostró ser más citada en El Salvador $56.5 \%$ y menos en Jamaica 31.3\%. La razón “le ayuda a sentir más confianza o ser más capaz de hablar con las personas en una situación social" presentó el nivel más alto en Brasil $42 \%$ y el más bajo en Chile 19.5\%. La razón "le ayuda a desinhibirse" presentó el valor más alto en Brasil 58.0\% y el más bajo en El Salvador 21.7\%. La razón "le ayuda a mantenerse bien cuando sale en la noche con sus amigos" presentó la mas alta mención en Nicaragua $45.8 \%$ y la menor en Chile $42.0 \%$. Por último, la razón "para relacionarse mejor con los demás debido a la presión social" fue mas reportada en El Salvador $17.4 \%$ y menos en Chile $7.3 \%$.

Tabla 4 - Razones asociadas al policonsumo simultáneo de sustancias psicoativas: fines sociales e implicaciones legales, según país, 2009-2010

\begin{tabular}{|c|c|c|c|c|c|c|c|c|c|c|}
\hline \multirow{3}{*}{ País/lugar } & \multicolumn{10}{|c|}{ Fines sociales } \\
\hline & \multicolumn{2}{|c|}{$\begin{array}{l}\text { Disfrutar com- } \\
\text { pañía }\end{array}$} & \multicolumn{2}{|c|}{ Más confianza } & \multicolumn{2}{|c|}{ Desinhibirse } & \multicolumn{2}{|c|}{$\begin{array}{l}\text { Mantenerse } \\
\text { bien }\end{array}$} & \multicolumn{2}{|c|}{$\begin{array}{l}\text { Relacionarse } \\
\text { mejor }\end{array}$} \\
\hline & $\mathrm{n}$ & $\%$ & $\mathbf{n}$ & $\%$ & $\mathbf{n}$ & $\%$ & $\mathbf{n}$ & $\%$ & $\mathbf{n}$ & $\%$ \\
\hline Brasil/Santo André $n=50$ & 26 & 52.0 & 21 & 42.0 & 29 & 58.0 & 21 & 42.0 & 8 & 16.0 \\
\hline Chile/Santiago $n=41$ & 18 & 43.9 & 8 & 19.5 & 11 & 24.4 & 10 & 24.4 & 3 & 7.3 \\
\hline Colombia/Cundinamarca $n=326$ & 118 & 36.2 & 66 & 20.2 & 77 & 23.6 & 95 & 29.1 & 29 & 8.9 \\
\hline Jamaica/Kingston $n=16$ & 5 & 31.3 & 4 & 25.0 & 6 & 37.5 & 4 & 25.0 & 2 & 12.5 \\
\hline Nicaragua/León $n=48$ & 18 & 37.5 & 13 & 27.1 & 13 & 27.1 & 22 & 45.8 & 7 & 14.6 \\
\hline El Salvador/San Salvador $n=23$ & 13 & 56.5 & 5 & 21.7 & 5 & 21.7 & 10 & 43.5 & 4 & 17.4 \\
\hline \multicolumn{11}{|c|}{ Implicaciones legales y policonsumo simultáneo de sustancias psicoativas } \\
\hline & \multicolumn{2}{|c|}{$\begin{array}{l}\text { Víctima de ac- } \\
\text { tos violentos }\end{array}$} & \multicolumn{2}{|c|}{$\begin{array}{c}\text { Tener acciden- } \\
\text { tes automovilís- } \\
\text { ticos }\end{array}$} & \multicolumn{2}{|c|}{$\begin{array}{l}\text { Ser lesionado } \\
\text { por otras per- } \\
\text { sonas }\end{array}$} & \multicolumn{2}{|c|}{$\begin{array}{l}\text { Problemas con } \\
\text { admón. Univer- } \\
\text { sitaria }\end{array}$} & \multicolumn{2}{|c|}{$\begin{array}{l}\text { Problemas con } \\
\text { la policía }\end{array}$} \\
\hline Brasil/Santo André $n=50$ & 8 & 16.0 & 3 & 6.0 & 3 & 6.0 & 5 & 10.0 & 2 & 4.0 \\
\hline Chile/Santiago $n=41$ & 3 & 7.3 & 6 & 14.6 & 2 & 4.9 & 3 & 7.3 & 1 & 2.4 \\
\hline Colombia/Cundinamarca $n=326$ & 28 & 8.6 & 15 & 4.6 & 21 & 6.4 & 22 & 6.7 & 17 & 5.2 \\
\hline Jamaica/Kingston $n=16$ & 4 & 25.0 & 4 & 25.0 & 6 & 37.5 & - & - & - & - \\
\hline Nicaragua/León n=48 & 11 & 22.9 & 6 & 12.5 & 6 & 12.5 & 23 & 47.9 & 11 & 22.9 \\
\hline El Salvador/San Salvador $n=23$ & 3 & 13.0 & 6 & 26.1 & 2 & 8.7 & 5 & 21.7 & 1 & 4.3 \\
\hline
\end{tabular}

\section{Implicaciones legales y policonsumo simultáneo de sustancias psicoativas}

Las implicaciones legales asociadas al policonsumo simultaneo (ver Tabla 4) mas citadas en Jamaica fueron "actos violentos" $25.0 \%$, "accidentes automovilísticos" $25.0 \%$, y "ser lesionado por otras personas" $37.5 \%$. En Nicaragua se observaron las menciones mas frecuentes de "problemas con la administración universitaria" $47.9 \%$ y "problemas con la policía" $22.9 \%$.

\section{Entornos asociados al policonsumo simultáneo}

Observamos que tres entornos aparecieron como asociados, de manera positiva o negativa, con el policonsumo simultáneo de sustancias psicoactivas. Estos fueron: estudiantil, familiar y de comportamiento sexual (datos no mostrados).
Para los estudiantes de Brasil, Colombia, Nicaragua y El Salvador las fiestas fueron importantes, factor que también se menciona asociado al policonsumo simultaneo. El estrés por problemas económicos no fue de mención frecuente entre los estudiantes en las universidades en Chile, Jamaica y Nicaragua que refirieron policonsumo simultáneo. Lo mismo ocurrió con el estrés por tareas universitarias, de baja mención en las dos universidades de Colombia entre estudiantes que reportaron policoncumo. En cuanto al entorno familiar, la "buena relación con los padres desde que iniciaron la universidad" obtiene una importante mención como protector del policonsumo en Brasil, Jamaica, Nicaragua y El Salvador. Para los estudiantes de las universidades de Chile y Colombia, el hecho de considerar que las actividades académicas son importantes es un elemento protector mas importante. La relaciones 
sexuales inesperadas o sin protección fueron mas mencionadas por estudiantes que reportaron policonsumo simultaneo.

\section{DISCUSIÓN}

\section{Policonsumo de sustancias psicoactivas}

El principal patrón de policonsumo simultáneo identificado en el presente estudio, para todos los estudiantes que afirmaron policonsumo en los últimos 12 meses y en los últimos 30 días, en la mayoría de países a excepción de Jamaica, correspondió a la combinación de alcohol + tabaco. Le siguen las combinaciones de alcohol + marihuana; alcohol + tabaco + marihuana, y alcohol + drogas de prescripción, con diferencias entre los estudiantes de las universidades participantes. Estos hallazgos coinciden con lo reportado en estudios, ${ }^{12}$ que evidencian similares combinaciones de mayor frecuencia entre estudiantes del área de la salud. Existe evidencia que el consumo de alcohol, tabaco y marihuana, solos o combinados, es un factor de riesgo para la transición hacia el consumo de otras sustancias psicoactivas como cocaína, especialmente en jóvenes, lo cual ha sido definido en otros estudios como "gateway model" ${ }^{15}$

Los factores que se mostraron como relacionados al policonsumo simultáneo de sustancias psicoactivas, ya sea de manera positiva o negativa, pueden ser agrupados en tres entornos: estudiantil, familiar y de comportamiento sexual. Esto se relaciona al modelo descrito por la teoría Biopsico-social de Engel, ${ }^{16}$ que postula la asociación con las dimensiones espirituales y culturales.

Las fiestas aparecen mencionadas en relación al policonsumo simultáneo. Las fiestas universitarias son además mencionadas en otros estudios como un lugar común donde los estudiantes promueven el uso de sustancias psicoactivas para ayudar a manejar la diversión y el estrés. ${ }^{3}$

La baja mención de factores de estrés económico y académico entre estudiantes que refirieron policonsumo podria reflejar resiliencia por parte de ellos, entendida como la adaptación exitosa ante la exposición a estresores significativos u otros factores que normalmente se considerarían de riesgo. ${ }^{17}$

Otro factor que no sorprende al ser señalado como protector del policonsumo simultáneo entre los estudiantes encuestados fue "la buena relación con los padres desde que iniciaron la universidad". Los alumnos que afirmaron haber tenido relacio- nes sexuales inesperadas o sin protección también refirieron con mayor frecuencia el policonsumo simultaneo, lo cual podria estar relacionado. Esta actitud riesgosa frente al sexo es mencionada en un estudio realizado en una escuela de Nueva York, en donde se reporta que el $25 \%$ de los estudiantes admitió incurrir en comportamiento sexuales riesgos mientras consumían sustancias psicoactivas. ${ }^{18}$

\section{CONCLUSIONES}

El principal patrón de policonsumo simultáneo identificado, en los últimos 12 meses y en los últimos 30 días, fue para la combinación de las sustancias psicoactivas alcohol + tabaco. Otras combinaciones frecuentes fueron: alcohol + marihuana; alcohol + tabaco + marihuana, y alcohol + drogas de prescripción.

Las razones mencionadas como asociadas al policonsumo simultaneo se pueden agrupar en el entorno estudiantil, familiar y del comportamiento sexual.

\section{Limitaciones}

La omisión en las respuestas pudiera introducir sesgos en el estudio. Los resultados de esta investigación no pueden ser generalizables a la población de estudiantes de las universidades participantes, ni a los países en donde están ubicadas, debido a que los participantes no constituyen un grupo representativo de la población estudiantil en general ni menos aun de la población joven, puesto que solo se consideraron los estudiantes de primer y segundo año de las Facultades de Ciencias de la Salud/Ciencias Médicas. La baja casuística identificada en algunas universidades participantes en el estudio, con relación a los estudiantes policonsumidores simultáneos, impidió la aplicación de modelos de inferencia estadística entre las variables estudiadas y pudo introducir potenciales sesgos en el estudio.

\section{Recomendaciones}

\section{A las universidades}

Realizar campañas informativas que permitan a los estudiantes conocer los factores de riesgo y los factores protectores asociados al policonsumo simultaneo de sustancias psicoactivas. Promover la organización de grupos conformados por estudiantes, familias y pares, que propendan 
por la reflexión, la discusión y el dialogo acerca de la problemática relacionada con el consumo de sustancias psicoactivas. Organizar actividades relacionadas con la vinculación a la vida universitaria para los estudiantes de primer año, con el fin de facilitar el proceso de transición de la vida escolar a la universitaria. Promover el desarrollo de las consejerías universitarias para los grupos de estudiantes más vulnerables a través de las asociaciones estudiantiles, así como el desarrollo de políticas universitarias que promuevan estilos de vida saludable.

\section{A las familias}

Estimular la creación de espacios que promuevan el conocimiento, la reflexión y el dialogo con los padres en torno a temáticas relacionadas con la dinámica universitaria y el fenómeno de las drogas.

\section{A las comisiones nacionales de drogas}

Gestionar ante las directivas de las universidades la incorporación del tema del fenómeno de las drogas, como parte del componente curricular a nivel de pre y postgrado. Gestionar recursos económicos en conjunto con las universidades y otros entes del estado, para promover el desarrollo de diferentes eventos de carácter académico en donde la temática sea el fenómeno de las drogas.

\section{A la CICAD/OEA}

Continuar el desarrollo de los procesos de capacitación de docentes y académicos en el campo de las drogas y las adicciones.

Promover el desarrollo de redes de investigación entre universidades, para la generación de conocimiento, así como la participación en foros de discusión y el desarrollo de programas de intercambio docente relacionados en esta temática, a fin de fortalecer la gestión con relación a la prevención del consumo y la promoción de estilos de vida saludables.

Sobre la base de cada uno de los modelos explicativos de los países participantes, se recomienda que cualquier intervención que tenga como objetivo el disminuir la incidencia del consumo simultáneo de sustancias psicoactivas entre los estudiantes de los primeros y segundos años de las facultades de ciencias médicas, se sugiere tener presente los siguientes aspectos; focalizarse en los aspectos que tengan que ver con el núcleo familiar, como es el hecho de no tener buenas relaciones con su padres y haber tenido algún tipo de problemas con su pareja sentimental, comportamiento sexuales tales como; haber tenido relaciones sexuales inesperada o sin protección asimismo enfocarse en los aspectos de la vida estudiantil, es decir, dirigir las intervenciones en aquellos estudiantes que se ausenten de clase con mucha frecuencias o que tengan poco interés en las actividades académicas.

\section{AGRADECIMIENTO}

Al Gobierno de Canadá/DFAIT, la Organización de Estados Americano (OEA), la Secretaría de Seguridad Multidimensional, la Comisión Interamericana para el Control del Abuso de Drogas (CICAD), al Centro de Adicciones y Salud Mental (CAHM/Toronto/Canadá), a todos los estudiantes por participar en el estudio, al Lic. Marlon Melendez por el análisis estadístico, finalmente a las autoridades universitarias por su apoyo y colaboración para con este estudio de los diferentes países participantes. Un agradecimiento especial al Dr. Gustavo Mery, CAMH Reviewer, por su colaboración en la revisión y editoración final de todos los trabajos y informes del grupo III.

\section{REFERENCIAS}

1. Laranjo T, Soares C. University residence halls: socialization processes and drug consumption. Rev Saude Pública. 2006 Dec; 40(6):1027-34.

2. Simons J, Gaher R, Correia C, Hansen C, Christopher M. Anaffective-motivational model of marijuana and alcohol problems among college students. Psychol Addict Behav. 2005 Sep; 19(3):326-34.

3. Pillon S, O'Brien B, Chavez K. The relationship between drugs use and risk behaviors in brazilian university students. Rev Latino-am Enfermagem. 2005; 13(Spe2):1169-76.

4. Giraldo H, Dennis R, Solarte I, Jurado A. Molina D. Vera A. Prevalencia de tabaquismo y sus factores asociados en adolescentes escolarizados de siete ciudades colombianas 2005-2006. Resumen ejecutivo. Bogotá (CO): Asociación Colombiana de Medicina Interna, Sociedad Colombiana de Neumología y Cirugía de Tórax, Sociedad Colombiana de Cardiología, Sociedad Colombiana de Oncología, Sociedad Colombiana de Pediatría. Informe sin publicar; 2006.

5. Organización de los Estados Americanos, Comisión Interamericana para el Control del Abuso de Drogas. Chile: Evaluación del Progreso de Control de Drogas, 2005-2006. Mecanismo de Evaluación Multilateral. Washington D.C. (US): OEA, CICAD; 2006. 
6. Organización de los Estados Americanos, Comisión Interamericana para el Control del Abuso de Drogas. El Salvador: Evaluación del Progreso de Control de Drogas, 2005-2006. Mecanismo de Evaluación Multilateral. Washington D.C. (US): OEA, CICAD; 2006.

7. Organización de los Estados Americanos, Comisión Interamericana para el Control del Abuso de Drogas. Jamaica: Evaluación del Progreso de Control de Drogas, 2005-2006. Mecanismo de Evaluación Multilateral. Washington D.C. (US): OEA, CICAD; 2006.

8. Organización de los Estados Americanos, Comisión Interamericana para el Control del Abuso de Drogas. Nicaragua: Evaluación del Progreso de Control de Drogas, 2005-2006. Mecanismo de Evaluación Multilateral. Washington D.C. (US): OEA, CICAD; 2006.

9. Panamerican Health Organization, World Heathl Organization. Estudio multicentrico sobre consume de alcohol, cultura y genero. Washington D.C. (US): PHO, WHO; 2006.

10. Huyse F, Lyons J, Stiefel F, Slates J, Jonge P, Latour C. Operationalizing the byosuchosocial model: the intermed. Psychosomatics. 2001 Jan-Feb; 42(1):5-13.

11. McCabe SE, Cranford JA, Morales M, Young A. Simultaneous and concurrent polydrug use of alcohol and prescription drugs: prevalence, correlates, and consequences. J Stud Alcohol. 2006 Jul; 67(4):529-37.
12. Barrett SP, Darredeau C, Pihl RO. Patterns of simultaneous polysubstance use in using university student. Hum Psychopharmacol. 2006 Jun; 21(4):25563.

13. Hernández R, Fernández C, Baptista P. Metodologia de la investigacion en ciencias sociales. $4^{\mathrm{a}}$ ed. Mexico D.F: McGraw-Hill; 2008.

14. Carvalho P, Casenave A, Barbosa E, Montoya E, Chaname E. Oliveira $\mathrm{H}$, et al. University students' perceived norms about peers and drug use: a multicentric study from five Latin Americ countries: research proposal. Washington D.C. (US)OASCICAD-CAMH; 2007.

15. Hall W, Lynskey M. Is cannabis a gateway drug? Testing hypotheses about the relationship between cannabis use and the use of other illicit drugs. Drug Alcohol Rev. 2005 Jan; 24(1):39-48.

16. Engel G. The need for a new medical model: a challenge for biomedicine. Science. 1977 Apr 8; 196(4286):129-36.

17. Becoña IE, Varela CM, López DA, Vazquez DJ, Pontevedra CL. Resilencia y consumo de alcohol en jóvenes. Salud y Drogas. 2006; 6(1):89-111.

18. Perkins HW. Surveying the damage: a review of research on consequences of alcohol misuse in college populations. J Stud Alcohol Suppl. 2002; (14):91-100. 